\title{
Envisioning Future Mobile Spatial Applications
}

\author{
Peter Fröhlich, Rainer Simon, Elisabeth Muss, Andrea Stepan, Peter Reichl \\ Telecommunications Research Center Vienna (ftw.) \\ Donau-City-Str.1 \\ 1220 Vienna, Austria \\ $+43 / 1 / 5052830-85$
}

\{froehlich, simon, muss, stepan, reichl\}@ftw.at'

\begin{abstract}
This paper presents two empirical investigations of future applications of mobile spatial interaction, i.e. the use of mobile phones as pointers to the real world. In situated interviews and a photo diary study, real-world objects of interest for referencing services were identified. Furthermore, envisioned services, their attractiveness and relevant usage situations were explored. The presented results of the study indicate that access to background information on buildings as well as spatiallyrelated search and service access are highly attractive for future users, whereas spatially-related purchase, advertisement, gaming, and sharing is of less interest..
\end{abstract}

\section{Categories and Subject Descriptors}

H.5 Information Interfaces and Presentation (I.7); H5.2 User Interfaces: User-Centered Design.

\section{General Terms}

Measurement, Design, Economics, Experimentation, Human Factors.

\section{Keywords}

Mobile Spatial Interaction, Future applications, Innovation, Empirical user studies

\section{INTRODUCTION}

Ongoing advances in hardware and software technology are currently enabling researchers to develop new ways of interacting with digital information. One of the most intriguing ideas is 'mobile spatial interaction', i.e. to use your mobile phone as a pointing device to real world objects (see [1] for a detailed overview).

The Point-to-Discover project (p2d, [3], see Fig. 1) aims at realizing this vision by means of a combination of GPS, digital compass and tilt sensors. p2d is a joint academic/industry research project that explores the technologies behind this innovative concepts for how people might discover and access information about their immediate environment in the near future. Apart from technological challenges, namely hardware, platform and mobile client development, it is also a non-trivial task to identify suitable application scenarios.

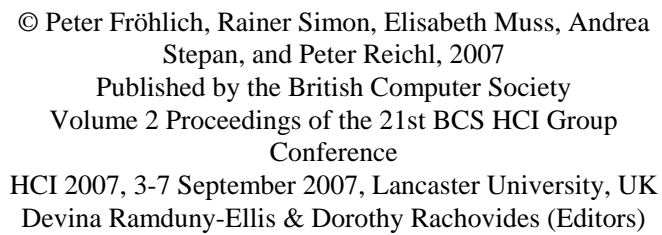

When exploring future use, it is essential to adopt an empirical, human-centered approach to innovation, rather than relying on design intuition or technology-driven thinking (compare [2]). As is the case with many ubiquitous research endeavors, it is hard to elicit meaningful and valid input from users, simply because the concepts are so new and uncommon. Moreover, it is impossible to investigate mobile spatial interaction concepts in standard stationary laboratories, due to the close relationship to spontaneous behavior in outdoor real world contexts.

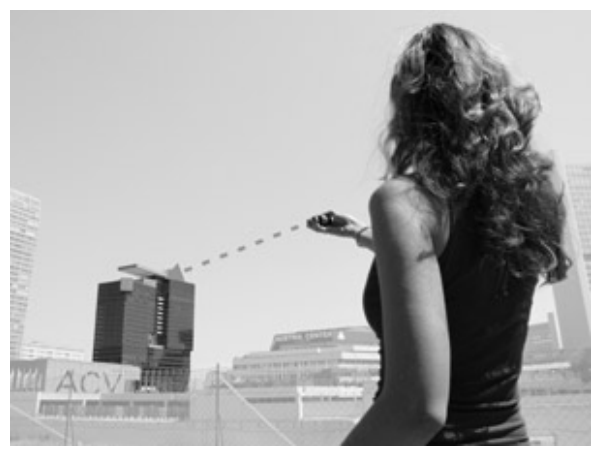

Figure 1. Accessing and annotating services referenced with surrounding objects: the p2d project

In our investigations we were addressing the following research questions:

1. How do people spontaneously envisage using $\mathrm{p} 2 \mathrm{~d}$ in a concrete setting? Which objects would they reference?

2. What would people like to do with p2d, i.e. which spatially-encoded services would they consider attractive?

3. In which situations would they be likely using p2d?

In the remainder of the paper, two user studies designed to find first answers to these questions are described in sections 2 and 3. Section 4 provides key results of both studies, which will then be discussed in section 5 .

\section{STUDY 1: SITUATED INTERVIEWS}

In this study, a moderator accompanied a test participant for a 40 minute walk along a specified route through Vienna's $4^{\text {th }}$ and $1^{\text {st }}$ district. The route was chosen to include different building topologies (e.g. narrow streets vs. large squares) and building occupancies (e.g. residential area, shopping street, market place vs. squares and boulevard with historic buildings).

The sample comprised 10 participants with a mean age of 32 years, and was balanced regarding sex and professional status (2 employees, 1 freelancer, 2 housewives, 4 students). Their Internet usage averaged about 12 hours per week. While all 
participants owned a mobile phone, 6 had not yet surfed in the Internet with a mobile phone, 3 occasionally, and 1 regularly ${ }^{1}$.

In the first part of the route, the participants were asked to spontaneously mention ideas related to objects in their surroundings. They were told to imagine that every surrounding object was already associated to information services. The participants were instructed to show each object they would use p2d for and to answer the questions summarized in Table 1.

Table 1. Questions asked for each referenced object in both user studies

\begin{tabular}{|c|c|}
\hline & Question \\
\hline 1 & Please name the object! \\
\hline 2 & Please briefly describe the object! \\
\hline 3 & $\begin{array}{c}\text { Which service(s) would you like } \\
\text { to use with regard to this object? }\end{array}$ \\
\hline 4 & $\begin{array}{c}\text { Please name situations in which the service } \\
\text { could be attractive! }\end{array}$ \\
\hline 5 & $\begin{array}{c}\text { Please provide a rating of the } \\
\text { attractiveness of the described service } \\
\text { (5-point rating scale)! }\end{array}$ \\
\hline
\end{tabular}

In the second part of the route, the participants were asked to imagine they could annotate content with p2d, for example: notes to buildings or other objects in form of a personal diary, to participate in a community or like in Flickr to take pictures that recognize the position and orientation. The rest of the instruction was the same as in the first part.

After having completed the route, an interview was conducted. Additionally to demographic questions, the participants were asked to rate the attractiveness of a set of service categories for p2d (see Table 2). They could again optionally name situations in which they considered the service attractive. Audio recordings were made throughout the course of the study, and the objects that the participants pointed at were photographed.

\section{STUDY 2: PHOTO DIARIES}

While the situated interviews aimed at capturing people's initial thoughts about p2d application possibilities, we were also interested in a longer-term observation. For this purpose, we conducted an 'imaginative diary study'. The main concept was to ask users to take photos of surrounding objects in their everyday life, for which they would like to use p2d.

Also here, the sample recruitment $(\mathrm{N}=11)$ aimed at a balanced distribution of age (mean: 34), sex (5 female; 6 male) and professional status. The average time actively spent on the Internet was 12 hours per week. All persons owned a mobile device, however only 2 had already experiences with the mobile Internet.

To account for different contexts, motivations and daily procedures, the participants' background was varied: 2 tourists, 3 students during their semester, 1 teenager during school period, 1 person on a business trip, 2 commuters, 1 housewife, and 1 retired. All participants owned and frequently used a digital camera.

[1]

${ }^{1}$ The sample composition deliberately accounted for the low usage rate of mobile Internet in the Austrian/European population in 2007.
There were 3 phases: a briefing workshop, the trial phase, and the final interview. In the briefing workshop the participants were informed about p2d and its general application potential (based on the service categories shown in Table 2) and about the further course of the study.

For the following 2 weeks, they were asked to take about 30 pictures of objects they would be interested to reference with p2d. They were provided with a diary template, in which they could annotate their digital photographs, following the same question structure as in Study 1 (see Table 1). A follow-up call at the end of the first week aimed at monitoring the status of the participants' progress and, if needed, at reminding users to take photos. The final interview was conducted singularly with each participant, based on the photos and annotations in the diary, which had been sent to the moderator in advance.

Table 2. Service categories, serving as structure for the interviews in both studies

\begin{tabular}{|c|c|}
\hline Service Category & Example \\
\hline $\begin{array}{c}\text { Background information to } \\
\text { certain buildings or objects }\end{array}$ & Historical information \\
\hline Spatially-related search & $\begin{array}{c}\text { The next restaurant or the } \\
\text { next taxi stand }\end{array}$ \\
\hline $\begin{array}{c}\text { Spatially-related access to } \\
\text { information services }\end{array}$ & $\begin{array}{c}\text { Public transport schedules } \\
\text { or the program of the opera }\end{array}$ \\
\hline $\begin{array}{c}\text { Spatially-related } \\
\text { advertisement }\end{array}$ & $\begin{array}{c}\text { Pointing at McDonalds and } \\
\text { participating in } \\
\text { competitions }\end{array}$ \\
\hline Spatially-related purchasing & $\begin{array}{c}\text { Directly order special offers } \\
\text { of a supermarket or find out } \\
\text { the price of real estates }\end{array}$ \\
\hline Spatially-related games & $\begin{array}{c}\text { Find people with similar } \\
\text { interests and know where } \\
\text { they are, or for paper-chase }\end{array}$ \\
\hline Spatially-related sharing & $\begin{array}{c}\text { A mobile diary with } \\
\text { comments to certain objects } \\
\text { or a forum }\end{array}$ \\
\hline
\end{tabular}

\section{RESULTS}

The results described in this section are structured along the three research questions formulated at the end of section 1 : object references, service attractiveness, and situational factors.

\subsection{Object reference}

\subsubsection{Information access}

In the situated interviews, an overall of 73 objects were mentioned as interesting pointing targets by the 10 participants. Only 2 of them were moving (tramway), the rest (71) were static. Most of the mentioned objects concerned commercial entities (47\%) such as shops, restaurants etc, followed by entire buildings (36\%) and others (19\%) such as signs, streets etc.

In the photo diary study, the 11 participants all in all selected 318 objects. Compared to the situated interview study, the proportion of entire buildings (48\%) was greater and the proportion of commercial entities (21\%) was smaller. Moreover, there was a greater share of other object references, for example public transportation means, posters and signs, natural landmarks, and public spaces (31\%, see a more detailed overview in Table 3). 
Table 3. Object types selected by the participants of the photo diary study

\begin{tabular}{|c|c|c|}
\hline Object type & Freq. & in \% \\
\hline Historical buildings (e.g. churches) & 53 & 16.7 \\
\hline Shops & 36 & 11.3 \\
\hline Institutions for sports or cultural activities & 34 & 10.7 \\
\hline Means of transport (public and private) & 24 & 7.5 \\
\hline Posters and signs & 19 & 6.0 \\
\hline Educational institutions & 17 & 5.3 \\
\hline Monuments & 16 & 5.0 \\
\hline Residential houses & 15 & 4.7 \\
\hline Landscape (e.g. rivers, mountains) & 14 & 4.4 \\
\hline Companies & 13 & 4.1 \\
\hline Restaurants & 13 & 4.1 \\
\hline Public places (e.g. market places and & 13 & 4.1 \\
\hline parks) & 10 & 3.1 \\
\hline Health care institutions & 8 & 2.5 \\
\hline Others & 6 & 1.9 \\
\hline Hotels & 27 & 8.5 \\
\hline
\end{tabular}

\subsubsection{Annotation}

The participants had a much harder time when instructed to add or annotate their surroundings in the second part of the situated interview study. They regarded only 36 objects suitable for annotation, in contrast to 73 objects for information access. Furthermore, in about half of the cases (19 of 36) they were more interested in passive usage of user-generated content, such as tourist fora or restaurant review services, not in making annotations themselves. The ideas concerning active annotations can be summarized as follows:

- Reviews and recommendations of restaurants and shops for others, e.g. "This book shop currently has amazing sales offers! Check it out!” (mentioned 7 times)

- Reminiscences for oneself, such as reminders to a good restaurant, or notes about interesting places one had enjoyed (mentioned 4 times).

- $\quad$ Reminders for future events ("I am interested in the event announced on THIS poster"; mentioned once).

- Co-ordination between friends (noting an attractive real estate offer, in order to discuss with a friend whether one should move in; mentioned once)

- Background information for others, perhaps comparable to a mobile Wikipedia (mentioned twice)

- Initiation of discussions about topics of local interest, e.g. "This is a never ending construction site! It's a shame that they mutilate this place for such a long time" (mentioned once).

- Gathering sound recordings from each place (brought up by a musician).

\subsection{Service attractiveness}

Service attractiveness was investigated in two ways. First, the participants had to state for each of the selected objects a specific service they would envision. During data analysis, the participants' ideas were allocated to the 7 service categories presented in Table 2. Fig. 2 summarizes the respective results for both studies.

The results from both studies are mostly similar in this regard. Most strikingly, spatially-related advertisement, gaming and sharing were not mentioned at all. The only notable difference was the higher prevalence of purchasing in the situated interview.

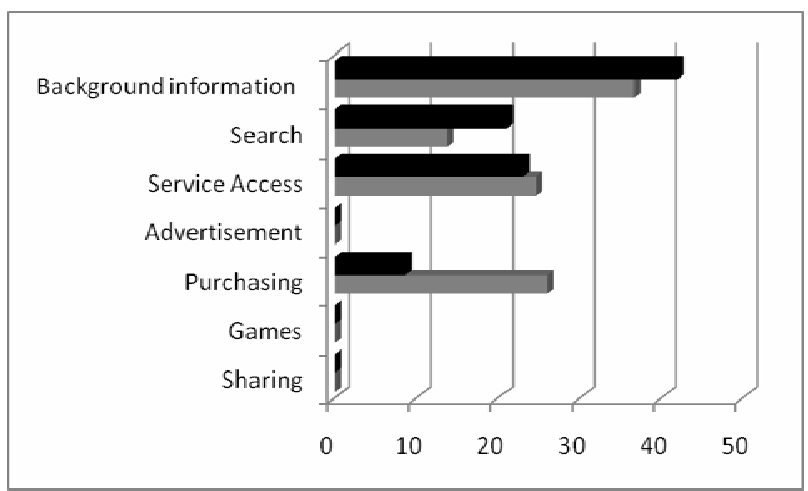

Figure 2. Percentage of $\mathbf{p} 2 \mathrm{~d}$ services referenced to objects. Grey bars represent situated interviews, black bars represent photo diary.

Second, in the final interviews of both studies, the participants directly provided attractiveness ratings for each of the 7 service categories. The results are presented in Fig. 3 below. Quite obviously there are two clusters of service categories: background information to buildings, spatially-related search and service access vs. spatially-related advertisement, purchasing, gaming, and sharing. Pairwise comparisons (Wilcoxon tests) between the service categories of the two clusters confirm this difference for both studies $(p<.05)$, with the exception that in the situated interview the attractiveness difference between spatially-related purchasing and service access did not reach significance $(\mathrm{p}<.065)$.

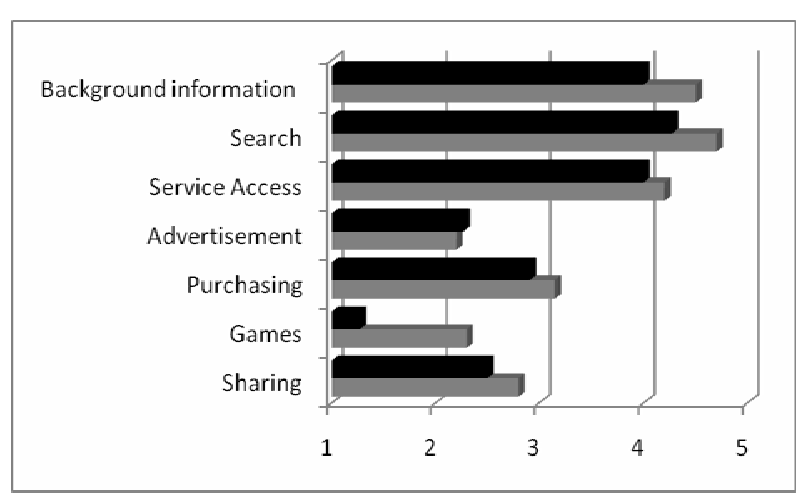

Figure 3. Attractiveness ratings for p2d service categories. Grey bars represent situated interviews, black bars represent photo diary. 


\subsection{Situational factors}

For each of the referenced objects, the participants could optionally mention situations, in which they would most likely use p2d for that purpose. During data analysis, the mentioned situations were classified into 5 categories. Fig. 4 presents the percentage-wise allocation to these categories. The huge difference between the two studies regarding the general category may be ascribed to a stronger motivation of giving an answer when being accompanied by an interviewer than when filling out a diary on her own.

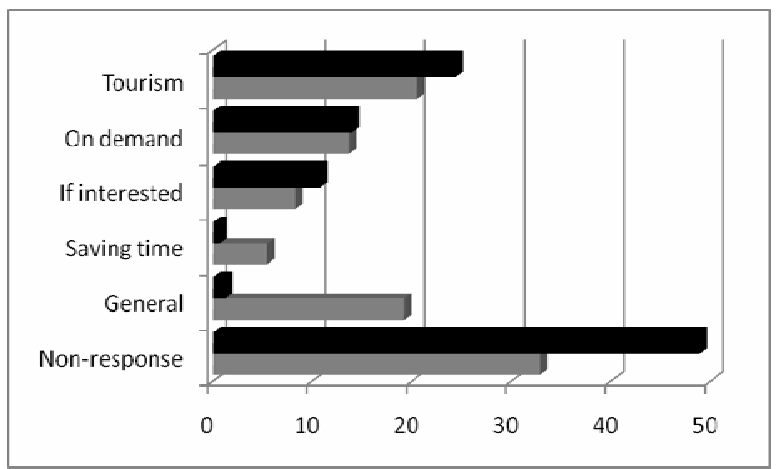

Figure 4. Percentage of situations mentioned during object referencing. Grey bars represent situated interviews, black bars represent photo diary.

\section{DISCUSSION AND CONCLUSIONS}

Two empirical studies have been conducted to explore the future use of mobile spatial interaction. The key results of both studies have shown to be consistent: the participants had strong interest in the - possibly more obvious and classic - uses of mobile spatial interaction (background information, search and service access), and were less comfortable with more advanced concepts, such as gaming, sharing, and also purchasing.

This finding is certainly due to the deliberate composition of a sample with an average openness to new technologies. We argue that, in order to envisage mass-market potential, user study samples should not have a strong bias towards earlyadopters.
Participants did not appear to have a strong need for annotating content to places by means of mobile devices. More efficient methods for input on mobile devices appear to be indispensable increase acceptance of such services.

The study results helped to inform and ground the p2d design and implementation process. Instead of an initially planned pervasive game, we decided to build a mobile guide and information service, both enabling mobile search and background information to buildings. In this way, our focus has been shifted from innovating complete application concepts towards innovating the access to conventional services by location and orientation awareness.

The qualitative user comments, which could only partly be reported in this paper, provide important insights and still require further analysis. A consequent next step of our empirical innovation process is to conduct user studies with functional p2d prototypes, in order to further refine the application scenario.

We hope that the methodological approach presented may be adopted in other ubiquitous computing research endeavors. It provides a way to achieve contextually meaningful results, with moderate use of resources. It is of course true that investigations of this style are highly sensitive with regard to country-specific or even regional usage habits and motivations. It would therefore be worthwhile to replicate these studies in other (non-European) countries.

\section{ACKNOWLEDGMENTS}

This work is part of the ftw. projects p2d and SUPRA, funded by Mobilkom Austria, Siemens Austria and the Austrian competence centre program Kplus.

\section{REFERENCES}

[1] Fröhlich, P., Simon, R., Baillie, L., Roberts, J., and Murray-Smith, R. (2007). Mobile Spatial Interaction. Extended Abstracts of CHI 2007. ACM Press. Workshop website: http://msi.ftw.at

[2] Oulasvirta, A. (2005). Grounding the innovation of future technologies. An Interdisciplinary Journal on Humans in ICT Environments. Volume 1 (1), April 2005, 58-75.

[3] Point-to-Discover project (p2d): http://p2d.ftw.at 